\title{
Properties Analysis of Lunar Regolith at Chang'E-4 Landing Site Based on 3D Velocity Spectrum of Lunar Penetrating Radar
}

\author{
Zejun Dong ${ }^{1,2}$, Xuan Feng ${ }^{1,2,3, *}$, Haoqiu Zhou ${ }^{1,2}{ }^{(}$, Cai Liu ${ }^{1,4}$, Zhaofa Zeng ${ }^{1,4}$, Jing Li $^{1,3}$ and \\ Wenjing Liang ${ }^{1,2}$ \\ 1 College of Geo-Exploration Science and Technology, Jilin University, No.938 Xi MinZhu Street, \\ Changchun 130026, China; dzj19@mails.jlu.edu.cn (Z.D.); zhouhq17@mails.jlu.edu.cn (H.Z.); \\ liucai@jlu.edu.cn (C.L.); zengzf@jlu.edu.cn (Z.Z.); inter_lijing@jlu.edu.cn (J.L.); liangwj@jlu.edu.cn (W.L.) \\ 2 Science and Technology on Near-Surface Detection Laboratory, Wuxi 214035, China \\ 3 Institute of National Development and Security Studies, Jilin University, No.2699 Qianjin Street, \\ Changchun 130012, China \\ 4 Key Laboratory of Geophysical Exploration Equipment, Ministry of Education (Jilin University), \\ No.938 Xi MinZhu Street, Changchun 130026, China \\ * Correspondence: fengxuan@jlu.edu.cn; Tel.: +86-431-8502258
}

Received: 18 January 2020; Accepted: 12 February 2020; Published: 13 February 2020

\begin{abstract}
The Chinese Chang'E-4 mission for moon exploration has been successfully completed. The Chang' $\mathrm{E}-4$ probe achieved the first-ever soft landing on the floor of Von Kármán crater $\left(177.59^{\circ} \mathrm{E}\right.$, $45.46^{\circ} \mathrm{S}$ ) of the South Pole-Aitken (SPA) basin on January 3, 2019. Yutu-2 rover is mounted with several scientific instruments including a lunar penetrating radar (LPR), which is an effective instrument to detect the lunar subsurface structure. During the interpretation of LPR data, subsurface velocity of electromagnetic waves is a vital parameter necessary for stratigraphic division and computing other properties. However, the methods in previous research on Chang' ${ }^{\prime} \mathrm{E}-3$ cannot perform velocity analysis automatically and objectively. In this paper, the 3D velocity spectrum is applied to property analysis of LPR data from Chang'E-4. The result shows that 3D velocity spectrum can automatically search for hyperbolas; the maximum value at velocity axis with a soft threshold function can provide the horizontal position, two-way reflected time and velocity of each hyperbola; the average maximum relative error of velocity is estimated to be $7.99 \%$. Based on the estimated velocities of 30 hyperbolas, the structures of subsurface properties are obtained, including velocity, relative permittivity, density, and content of $\mathrm{FeO}$ and $\mathrm{TiO}_{2}$.
\end{abstract}

Keywords: Chang'E-4; lunar penetrating radar (LPR); 3D velocity spectrum; properties analysis

\section{Introduction}

The Chinese Chang'E-4 mission for moon exploration has been successfully completed. The Chang'E-4 probe achieved the first-ever soft landing on the floor of Von Kármán crater $\left(177.59^{\circ} \mathrm{E}\right.$, $45.46^{\circ} \mathrm{S}$ ) of the South Pole-Aitken (SPA) basin on January 3, 2019 [1-3]. SPA basin is the broadest basin on the Moon. This ancient basin was born from asteroid impacts 4 billion years ago, recording the evolutionary history of the far side of the Moon, and is of great significance for researching the internal materials and structures of the Moon [4-6]. Von Kármán crater is one of the primary craters in the SPA basin, with a diameter of $186 \mathrm{~km}$. Recent studies have revealed that the ejecta from adjacent craters have various contributions to the subsurface material of Von Kármán crater, which results in the complex subsurface structure at the Chang'E-4 landing site [1,3,4]. 
In order to patrol and investigate the lunar surface, a Yutu-2 rover is carried by Chang'E-4 probe. Yutu-2 rover is mounted with several scientific instruments containing a lunar penetrating radar (LPR) which has been verified to be an effective device for detecting the lunar subsurface structure $[7,8]$. The LPR is equipped with two types of channels (CH-1 and $\mathrm{CH}-2)$, the center frequencies of which are $60 \mathrm{MHz}$ and $500 \mathrm{MHz}[9,10]$. In addition, the $\mathrm{CH}-2$ possesses one transmitting antenna and two receiving antennas of different offsets which are also known as $\mathrm{CH}-2 \mathrm{~A}$ and $\mathrm{CH}-2 \mathrm{~B}$ [10-12]. With different frequencies, $\mathrm{CH}-1$ and $\mathrm{CH}-2$ have different detecting missions. The objective of $\mathrm{CH}-1$ is to detect the deep structure of Von Kármán crater [13]; CH-2 is to map the detail of near surface layers, leading to the property analysis of lunar regolith $[14,15]$.

During the interpretation of LPR data, subsurface velocity of electromagnetic waves is a vital parameter necessary for stratigraphic division and computing other properties. However, the velocity analysis of field LPR data faces many difficulties. Complex subsurface structure and interference of noise always result in the incomplete, interlaced, and amplitude-varying hyperbolas. In previous studies of Chang'E-3, Feng et al. proposed hyperbolic fitting method for velocity analysis of LPR data [10]; Lai et al. used two-way delay method to acquire the velocity [16]; Zhang et al. applied $\mathrm{CH}-2 \mathrm{~A}$ and $\mathrm{CH}-2 \mathrm{~B}$ data of different offsets to estimate the velocity [17]. However, these methods need humans to select the hyperbolas, which is highly subjective. Thus, in this article, we applied 3D normalized velocity spectrum to estimate the velocity [18]. This method can automatically and objectively select hyperbolas and analyze the velocities; the normalization processing can solve the error brought by different amplitudes of different positions on hyperbolas; subsequently, during the computation, we applied a variable horizontal computation window along longitudinal direction to satisfy the field situation that rock sizes increase vertically.

This paper is organized as follows. Section 2 introduces basic theory of 3D velocity spectrum and properties analysis. In Section 3, firstly a model test is performed to verify the feasibility of 3D velocity spectrum; subsequently the method is applied to the CH-2B data analysis. Subsequently, based on the $3 \mathrm{D}$ velocity spectrum, we obtain the positions and velocities of the points where hyperbolas exist; then the property structures of lunar regolith are computed including velocity, relative permittivity, density, and content of $\mathrm{FeO}$ and $\mathrm{TiO}_{2}$. In Section 4, we discuss the computation error of each hyperbola. Section 5 is the conclusion.

\section{Methods}

\subsection{D Velocity Spectrum}

The velocity spectrum analysis method is originally based on common middle point (CMP) ground penetrating radar (GPR) data $[19,20]$, but it can also be applied to common offset data processing such as LPR data processing. In order to estimate velocity, a stacked amplitude is computed as follows:

$$
\begin{gathered}
S_{i, j, k}=\sum_{j=1}^{N_{\mathrm{i}}} f\left(t_{i, j, k}, x_{j}\right) \\
i=1, \ldots n t ; \quad j=1, \ldots n x ; \quad k=1, \ldots n v
\end{gathered}
$$

where $f$ is the LPR data in $t-x$ domain; $N_{i}$ denotes the selected horizontal computation region size at $i$ th time; to satisfy the field situation that rock sizes increase vertically, we make $N_{i}$ variations along longitudinal direction; $n t, n x$, and $n v$ are the number of sampling points of each trace, number of traces, and number of velocities used in computation; $x_{j}$ represents the horizontal distance between the $j$ th point and the extreme point of the hyperbola; $t_{i, j, k}$ is the two-way time of the $j$ th points of the hyperbola [21,22], which can be obtained using the formula below:

$$
t_{i, j, k}=\left(t_{i}^{2}+\frac{4 x_{j}^{2}}{v_{k}^{2}}\right)^{1 / 2}
$$


where $t_{i}$ is the two-way time of extreme point of the hyperbola; $v_{k}$ represents the velocity used in computation.

However, the method of (1) is not always effective in the field as the hyperbolas are incomplete, interlaced, with varying amplitude. To solve the error brought about by these situations, the normalization form of stacked amplitude is applied:

$$
C_{i, j, k}=\frac{1}{N_{i} L} \sum_{l=i}^{L+i-1}\left[\frac{S_{i, j, k}^{2}}{\sum_{j=1}^{N} f^{2}\left(t_{i, j, k}, x_{j}\right)}\right]
$$

where $L$ is the time gate; the average in the time gate is adopted because the hyperbolas on radargram are not curves but regions with hyperbolic shapes. The normalized form can guarantee that the result $C_{i, j, k}$ is not affected by the varying amplitude. Importantly, $1 / N_{i}$ is added in this form to compensate the energy differences caused by using different windows $N_{i}$ along longitudinal direction. $C_{i, j, k}$ is $3 \mathrm{D}$ data, whose local maximum values indicate the time, horizontal position, and velocity of the points with hyperbolas.

\subsection{Properties Computation}

After we obtain the velocities from the 3D spectrum, the properties can be computed. Without considering magnetic permeability, the relative permittivity can be easily derived using the following formula:

$$
\varepsilon=(c / v)^{2}
$$

where $c$ equals $0.3 \mathrm{~m} / \mathrm{ns}$.

According to the studies of lunar regolith samples of Apollo, there is a relation between the relative permittivity and density of lunar regolith [9,17]:

$$
\begin{gathered}
\rho=\log _{1.919}(\varepsilon) \\
\tan \delta=10^{0.440 \rho-2.943}
\end{gathered}
$$

The $\tan \delta$ is the loss tangent which represents the ratio of the imaginary part of the dielectric constant to its real part. The density of lunar regolith is shown in Figure 14. Subsequently, previous studies show that the loss tangent is also related to the $\mathrm{TiO}_{2}$ and $\mathrm{FeO}$ weight percentage values $[9,17]$ :

$$
\tan \delta=10^{\left[0.038 \times\left(\omega\left(\mathrm{TiO}_{2}\right)+\omega(\mathrm{FeO})\right)+0.312 \rho-3.26\right]}
$$

Combining (6) and (7), we can obtain the $\mathrm{TiO}_{2}$ and $\mathrm{FeO}$ weight percentage of lunar regolith.

\section{Results}

\subsection{Model Test}

Before the processing of LPR data, a model test was performed to verify the feasibility of 3D velocity spectrum. The relative permittivity model used in test and its simulated GPR profile are shown in Figure 1a,b, respectively. In the simulation, the frequency of electromagnetic wave is $100 \mathrm{MHz}$; the time window and the sampling interval are $100 \mathrm{~ns}$ and $0.32 \mathrm{~ns}$; the trace interval is $0.1 \mathrm{~m}$; the transmitting and receiving antennas are placed on the ground surface. The radargram shows there are three pairs of hyperbolas $(\mathrm{H} 1, \mathrm{H} 2$, and $\mathrm{H} 3$ ); the double hyperbolas generate from the upper and bottom surface of the scatters. Subsequently, the 3D velocity spectrum is computed, whose result is shown in Figure 1c. 
In fact, for a point $\left(t_{i}, x_{j}\right)$, there is only one true velocity; this true velocity corresponds to the maximum value along velocity axis; the maximum value can help to find the position of hyperbolas on $\left(t_{i}, x_{j}\right)$ profile. Thus, the maximum values $C_{i, j}$ along velocity axis are figured out using (8). Subsequently, in order to suppress the noise interference and obtain the accurate positions of hyperbolas, we apply a soft threshold function [23] as (9) shows. The value of $\varepsilon$ is set to 0.3 and the result is shown in Figure $1 \mathrm{~d}$. The energy stacks in three rectangles indicate the positions of hyperbolas. The irregular energy stack in the ellipse is generated by the intersection of two hyperbolas.

$$
\begin{gathered}
C_{i, j}=\max _{k} C_{i, j, k} \\
C_{i, j}= \begin{cases}C_{i, j}-\varepsilon & C_{i, j}>\varepsilon \\
0 & C_{i, j}<\varepsilon\end{cases}
\end{gathered}
$$

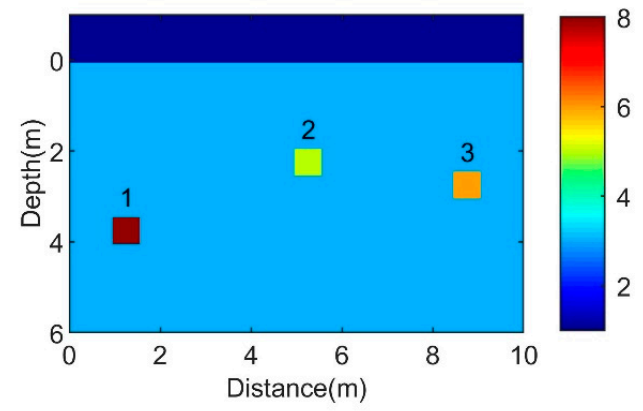

(a)

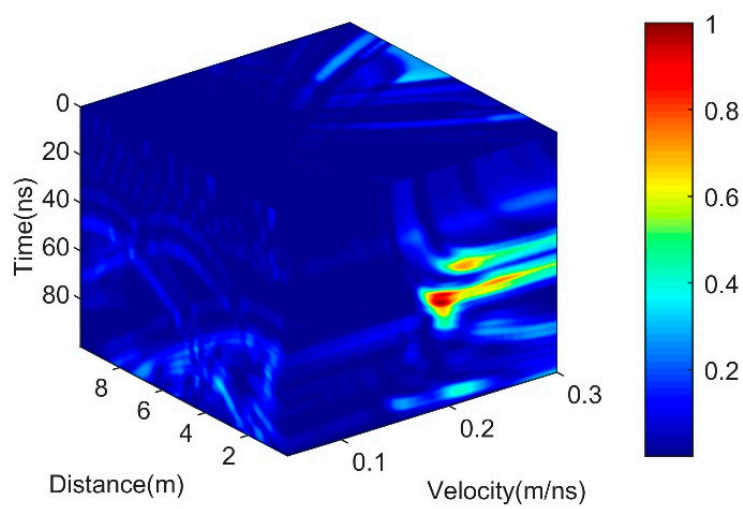

(c)

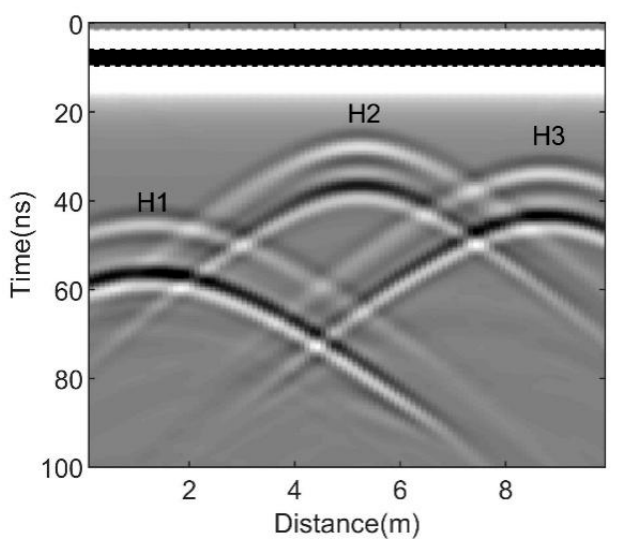

(b)

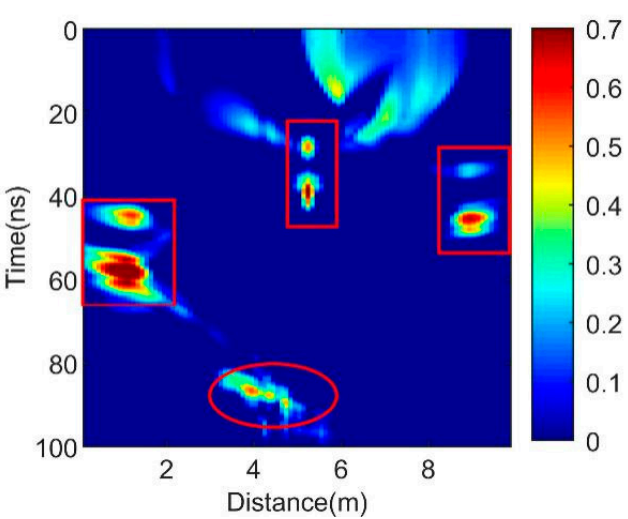

(d)

Figure 1. Model test results. (a) The model used in simulation; (b) the simulated GPR profile; (c) 3D velocity spectrum of (b); (d) maximum value along velocity axis with the soft threshold function processing.

After the $x$ position is located, the velocity spectrum slices can be selected out to estimate the velocities. The spectrum contour slices of the three hyperbolas are shown in Figure 2. From Figure 2, the velocities can be read out. The estimated results and errors are shown in Table 1. The results show that the estimation errors of upper hyperbolas are about $1 \%$, and the estimation errors of bottom hyperbolas are about $10 \%$. The errors are in acceptable range; the relatively higher errors of bottom hyperbolas result from the effects of scatters; the low velocities of electromagnetic waves inside scatters reduce the estimated velocities. 

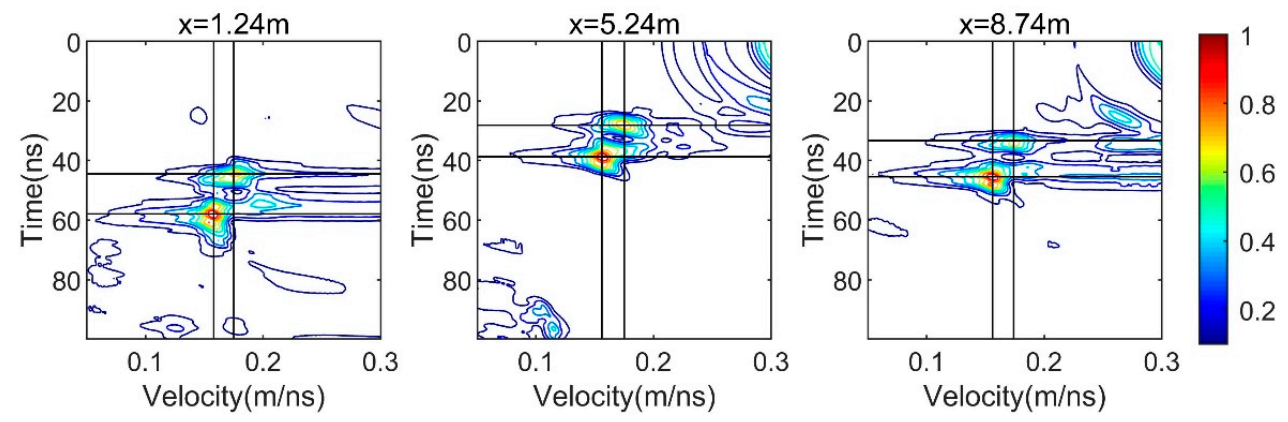

Figure 2. Velocity spectrum contour slices of three hyperbolas. The black crossed lines indicate the positions of maximum value.

Table 1. The estimated results and errors of model test.

\begin{tabular}{ccccc}
\hline \multirow{2}{*}{ Scatters } & \multicolumn{2}{c}{ Upper Hyperbolas } & \multicolumn{2}{c}{ Bottom Hyperbolas } \\
\cline { 2 - 5 } & Velocity (m/ns) & Error (\%) & Velocity (m/ns) & Error (\%) \\
\hline 1 & 0.175 & 1.04 & 0.158 & 8.78 \\
2 & 0.175 & 1.04 & 0.156 & 9.93 \\
3 & 0.174 & 0.46 & 0.156 & 9.93 \\
\hline
\end{tabular}

\subsection{Lunar Penetrating Radar (LPR) Data Processing}

The walking route of Yutu-2 rover for the first day and the second day on the Moon is shown in Figure 3. The length of the path is about $105 \mathrm{~m}$. Subsequently, the multi-segment data are spliced to achieve the $\mathrm{CH}-2 \mathrm{~B}$ profile. After a series of processing (Table 2) the LPR profile is obtained, which is shown in Figure 4.

Table 2. Details of data processing.

\begin{tabular}{|c|c|}
\hline Processing & Details \\
\hline Traces amending & $\begin{array}{c}\text { Adjusting the longitudinal displacement of traces, based on the phase of a strong } \\
\text { reflection event }\end{array}$ \\
\hline Traces selecting & $\begin{array}{l}\text { The rover might stop at some points on the way to collect other scientific data but LPR } \\
\text { never stops measurement, resulting in repeated acquisition of multiple traces at the same } \\
\text { location. We average the repeated traces. }\end{array}$ \\
\hline $\begin{array}{l}\text { Time lag } \\
\text { adjustment }\end{array}$ & $\begin{array}{c}\text { There is a } 28 \text { ns delay for the start time of the transmitting antenna compared to the } \\
\text { receiving antenna. }\end{array}$ \\
\hline $\begin{array}{l}\text { Useless data } \\
\text { deleting }\end{array}$ & Signals after $500 \mathrm{~ns}$ are removed since these signals are not reliable. \\
\hline $\begin{array}{l}\text { Attenuation compensation } \\
\text { Background removal }\end{array}$ & $\begin{array}{c}\text { Conducting automatic gain control (AGC) to make deep information more visible } \\
\text { Removing the average data along the rover path. }\end{array}$ \\
\hline $\begin{array}{l}\text { Band-pass } \\
\text { filtering }\end{array}$ & Adopting band-pass filtering to suppress the low-frequency and high-frequency noise. \\
\hline
\end{tabular}




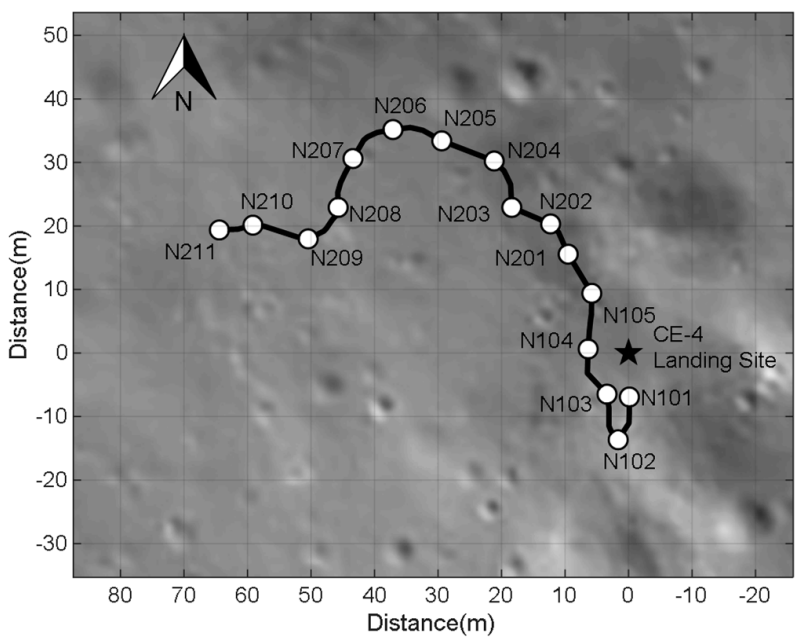

Figure 3. The walking route of Yutu-2 rover for the first day and the second day on the Moon.

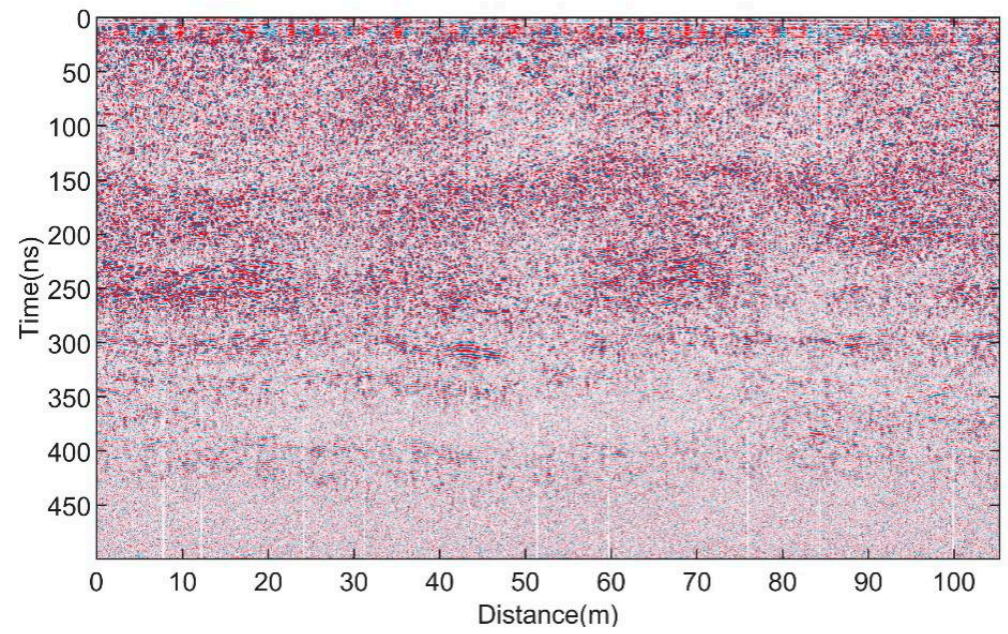

Figure 4. The $\mathrm{CH}-2 \mathrm{~B}$ profile of lunar penetrating radar (LPR) data.

\subsection{D Velocity Spectrum}

Based on (1)-(3), the 3D velocity spectrum is computed; the range of values are limited to 0 to 1 ; the $3 \mathrm{D}$ result without compensation of $1 / N_{i}$ is shown in Figure 5.

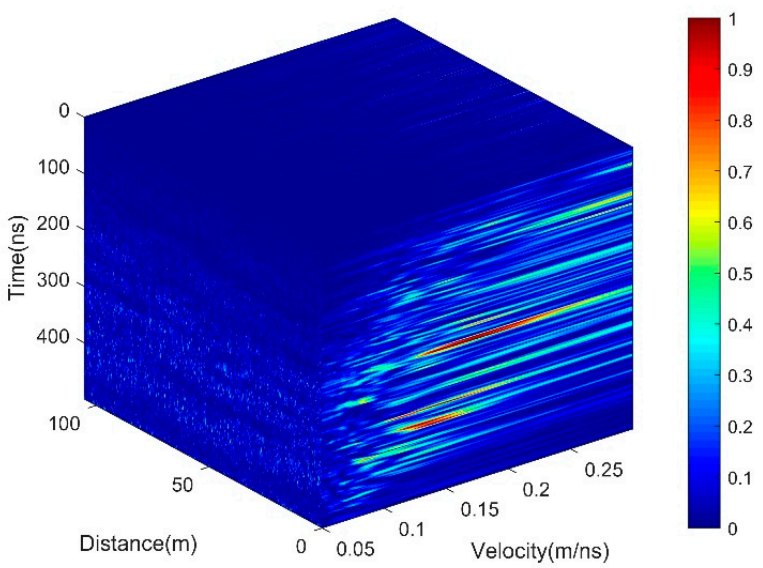

Figure $5.3 \mathrm{D}$ velocity spectrum. 
In order to locate the positions of hyperbolas on GPR profile, the maximum values $C_{i, j}$ along velocity axis are also figured out, which is shown in Figure 6a. Subsequently, 1/N $N_{i}$ is adopted to compensate the energy differences caused by using different windows $N_{i}$ along longitudinal direction, the result is shown in Figure $6 \mathrm{~b}$. The result shows the energies in shallow regions are compensated.

Subsequently, the soft threshold function is also applied. The value of $\varepsilon$ is set to 0.6. Subsequently, based on the result of soft threshold processing, the locations of local maximums are selected which are shown in Figure 7.

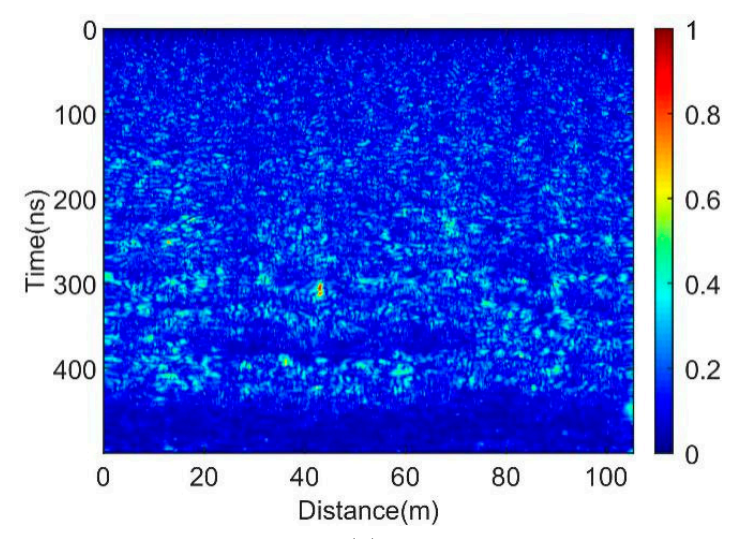

(a)

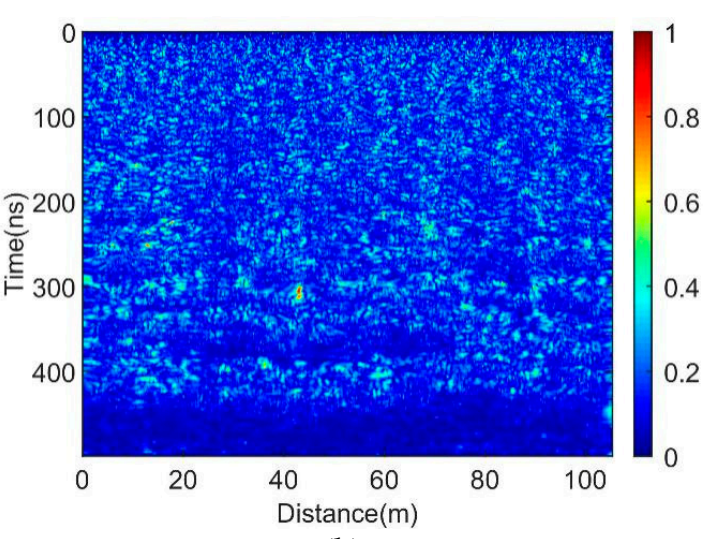

(b)

Figure 6. Maximum value along velocity axis. (a) Before compensation of $1 / N_{i} ;$ (b) after compensation of $1 / N_{i}$.

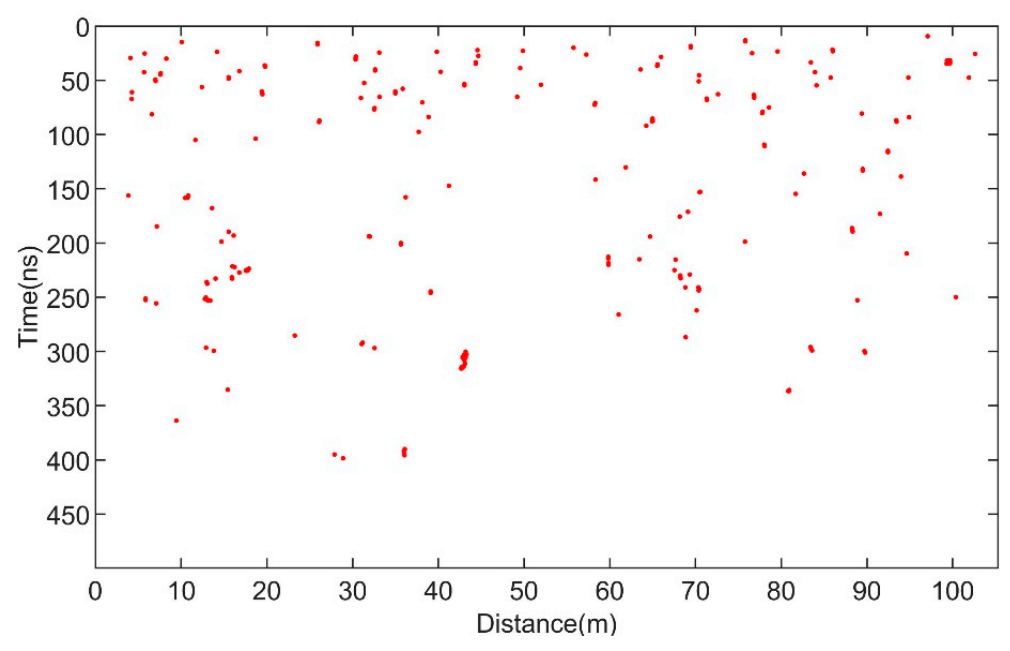

Figure 7. Initial positioning of hyperbolas based on soft threshold.

Based on Figure 7, we can search for the velocities in 3D velocity spectrum in Figure 5. However, not all the points in Figure 7 are useful, there are three reasons:

(1) For a highly flat interface, the estimated velocity will reach to $0.3 \mathrm{~m} / \mathrm{ns}$, which is obviously wrong; the lunar regolith velocity is less than $0.2 \mathrm{~m} / \mathrm{ns}$ [24], so we delete the points with velocities close to $0.3 \mathrm{~m} / \mathrm{ns}$.

(2) There may be a large stacked energy even if there are no hyperbolas but interlaced regions of several hyperbolas; for this situation, we should delete the non-hyperbolic points.

(3) The hyperbolas on radargram are not curves but regions with hyperbolic shapes, so there will be several excess points at one hyperbola, which should be deleted.

After filtering using the above method, the 30 preserved hyperbolas are noted in Figure 8a; the velocity spectrum slices of these hyperbolas are shown in Figure $8 \mathrm{~b}$. 


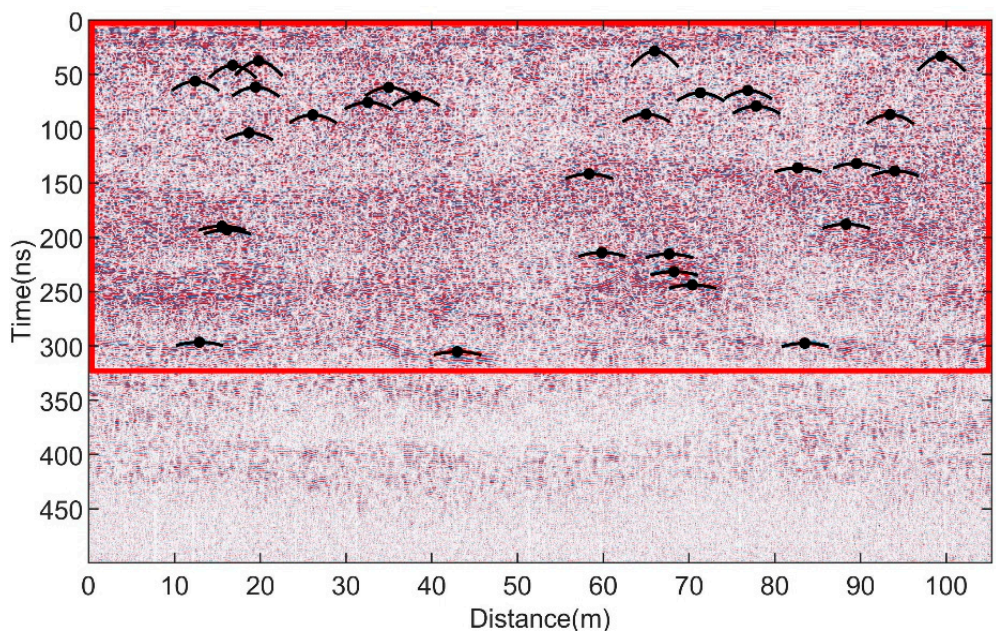

(a)
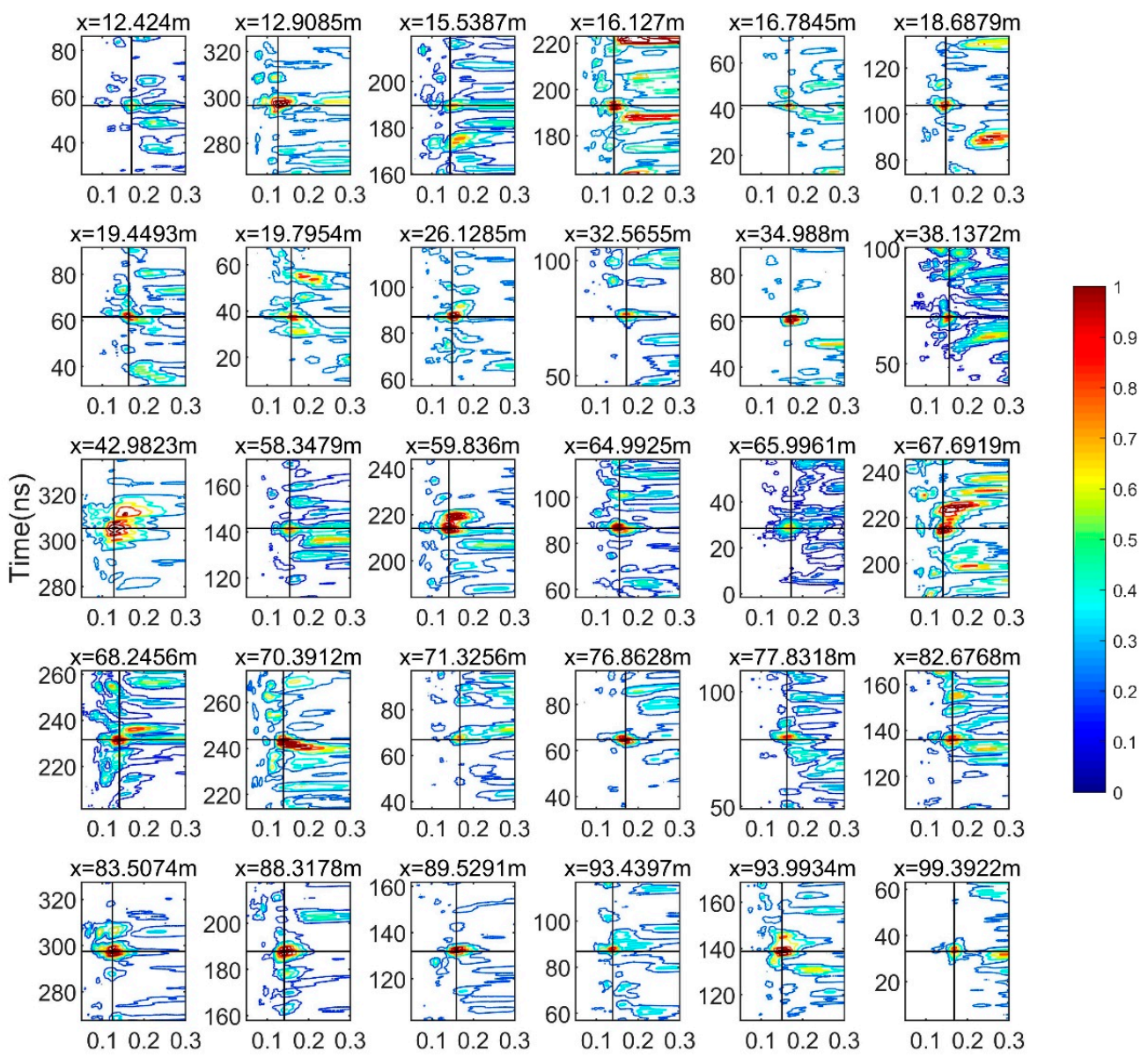

$\operatorname{Velocity}(\mathrm{m} / \mathrm{ns})$

(b)

Figure 8. Result of hyperbola selection. (a) Hyperbolas on radargram; (b) velocity spectrum slices of 30 hyperbolas - the black crossed lines indicate the positions of maximum values.

\subsection{Analysis of Radargram}

For Figure 8a, the hyperbolas located inside of the red rectangle, the region of 320-500 ns cannot be analyzed, so we focus on the region of 0-320 ns. Based on the reflection energy, several distinct layers and special regions are noted on the radargram (Figure 9). Layer 1 is a surface layer; a distinct reflection energy difference can be seen at $25 \mathrm{~ns}$. Layer 3 is the bottom layer; although its adjacent upper 
and lower areas show few reflections, the interface shows strong reflection energy. Layer 2 is a complex layer. The zone of 25-150 ns is relatively more uniform than the deeper zone; it contains few reflections indicating its high weathering degree. The zone of 150-300 ns is more complex possessing strong reflections; the complex signals are formed by the interlaced hyperbolas generated by the scattering of waves on rocks. Four obvious regions are selected out. The strong reflections within Regions 1 and 3 are distinct from the adjacent areas. The reflection energy in Region 2 is relatively lower than that of Regions 1 and 3, which means the material and horizontal structure changes between Regions 1 and 3. Region 4 also contains strong reflections, but its vertical size is shorter because the material and horizontal structure changes again at the position of $80 \mathrm{~m}$; the material at about $250 \mathrm{~ns}$ in Region 3 does not extend to Region 4. Overall, the subsurface material and structure at the Chang'E-4 landing site vary both vertically and horizontally, so the simple horizontal stratigraphic division is not appropriate within the zone of 25-300 ns.

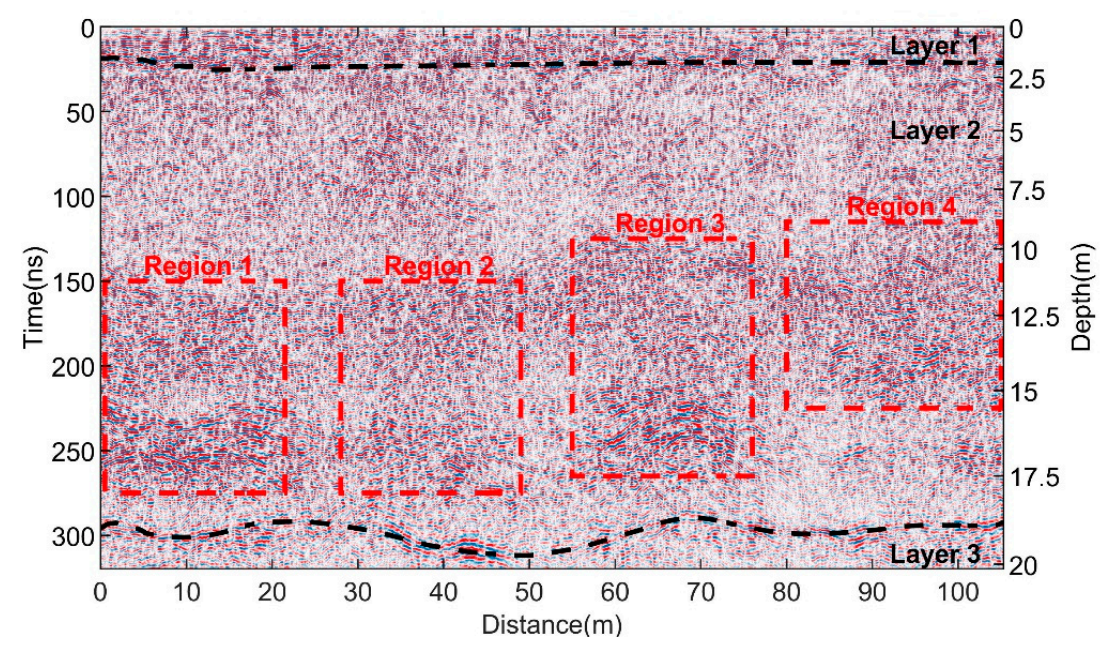

Figure 9. Analysis of radargram.

\subsection{Properties Analysis}

Subsequently, properties analysis is applied to LPR data. Firstly, we combine the positions and velocities of selected hyperbolas in Figure 8 and obtain a velocity scatter figure (Figure 10). The region size is the same with Figure 9, the color indicates the velocity of each point.

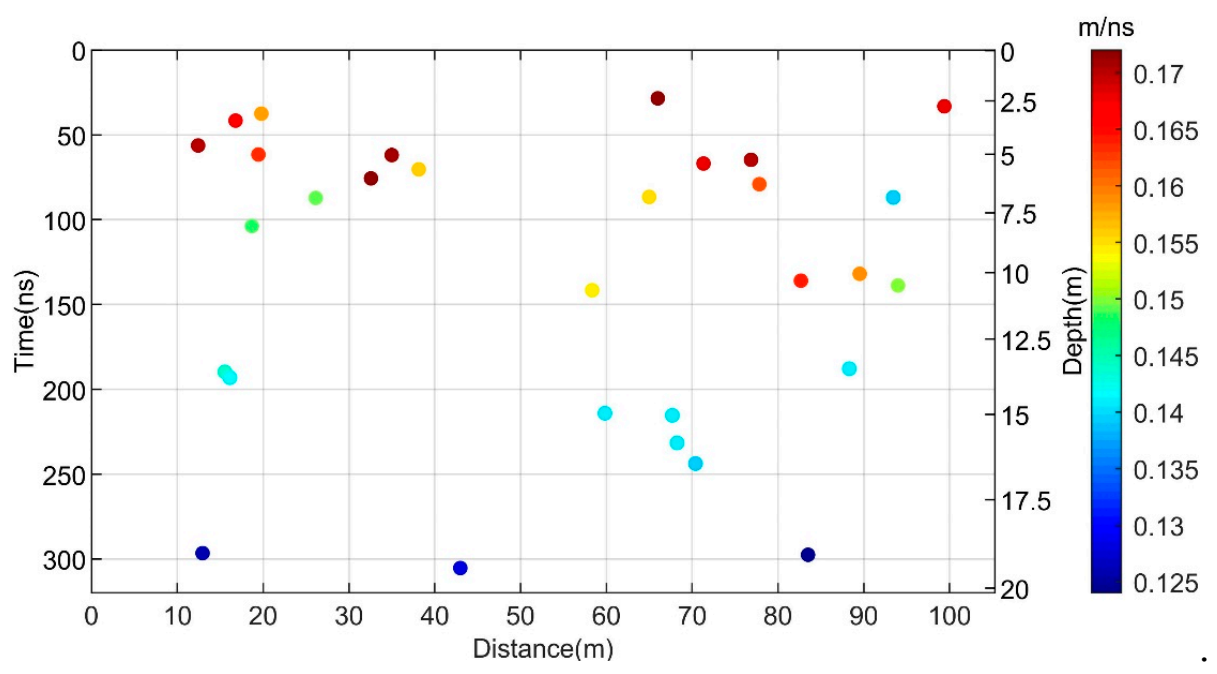

Figure 10. Velocity scatter figure. 
Subsequently, the image interpolation is applied to obtain the subsurface velocity of electromagnetic wave structure, then the smoothing is performed to remove the effects of outliers. Finally, as the velocities calculated by hyperbolic fitting method are the root-mean-square velocity (RMS), the interval velocities should be derived using the Dix formula:

$$
v_{i n t, n}=\sqrt{\frac{v_{r m s, n}^{2} t_{n}-v_{r m s, n-1}^{2} t_{n-1}}{t_{n}-t_{n-1}}}
$$

where $v_{i n t, n}$ and $v_{r m s, n}$ represent the interval velocity and root-mean-square velocity of $n$th layer respectively, $t_{n}$ is the travel time to the $n$th layer. By using (10), the subsurface interval velocity structure is computed, and the profile is shown in Figure 11. Subsequently, based on the velocities, the relative permittivity, density, and content of $\mathrm{FeO}$ and $\mathrm{TiO}_{2}$ can be derived using (4)-(7), the results are shown in Figures 12-14, respectively.

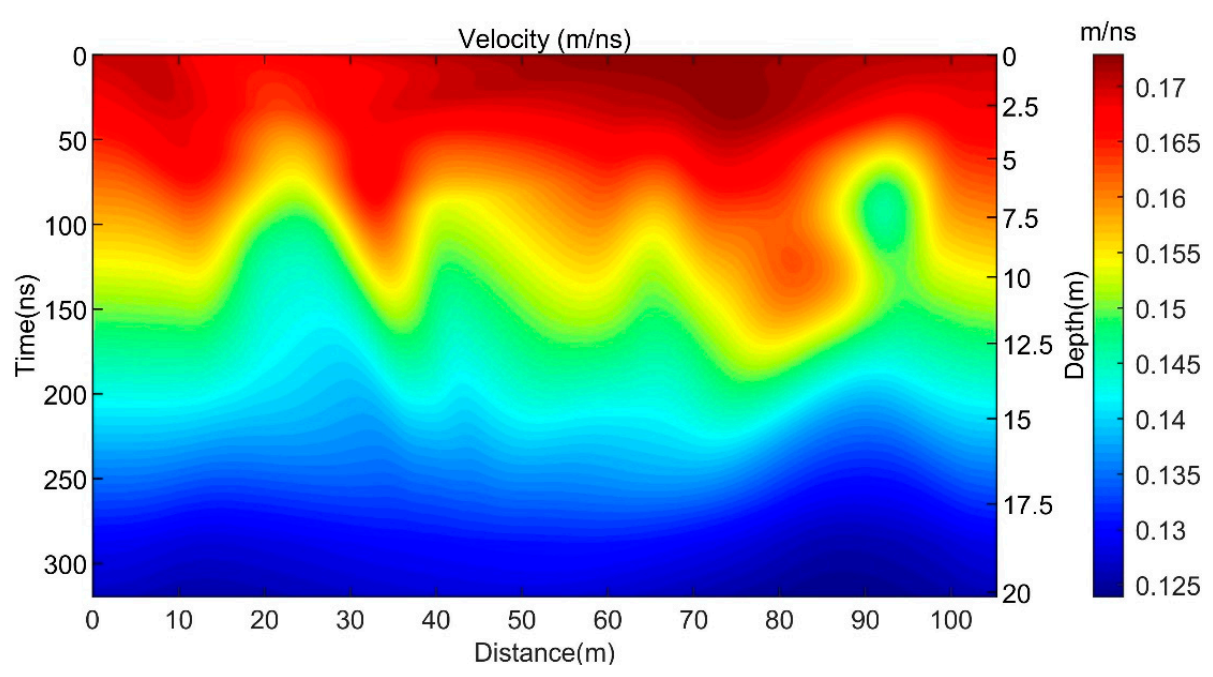

Figure 11. The subsurface electromagnetic wave velocity structure.

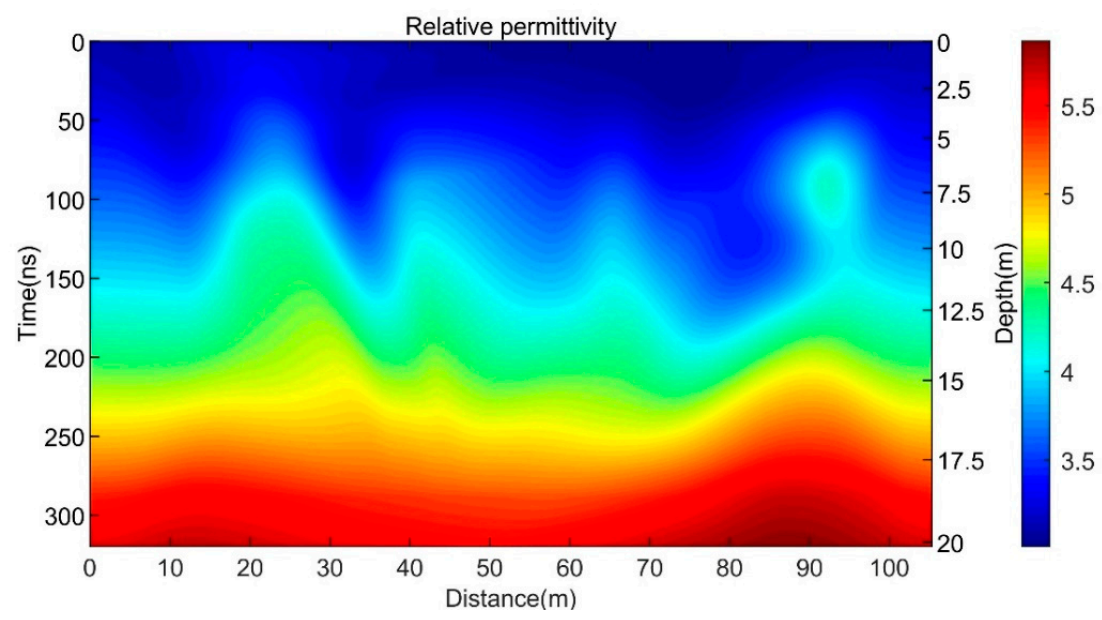

Figure 12. The subsurface relative permittivity structure. 


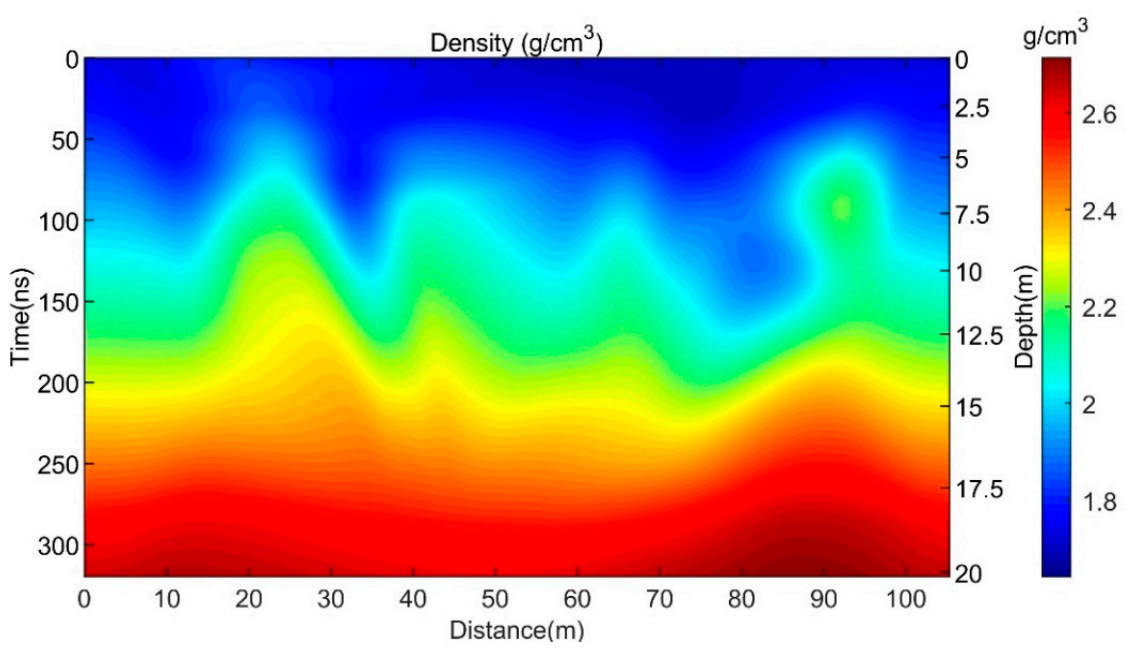

Figure 13. The density structure of lunar regolith.

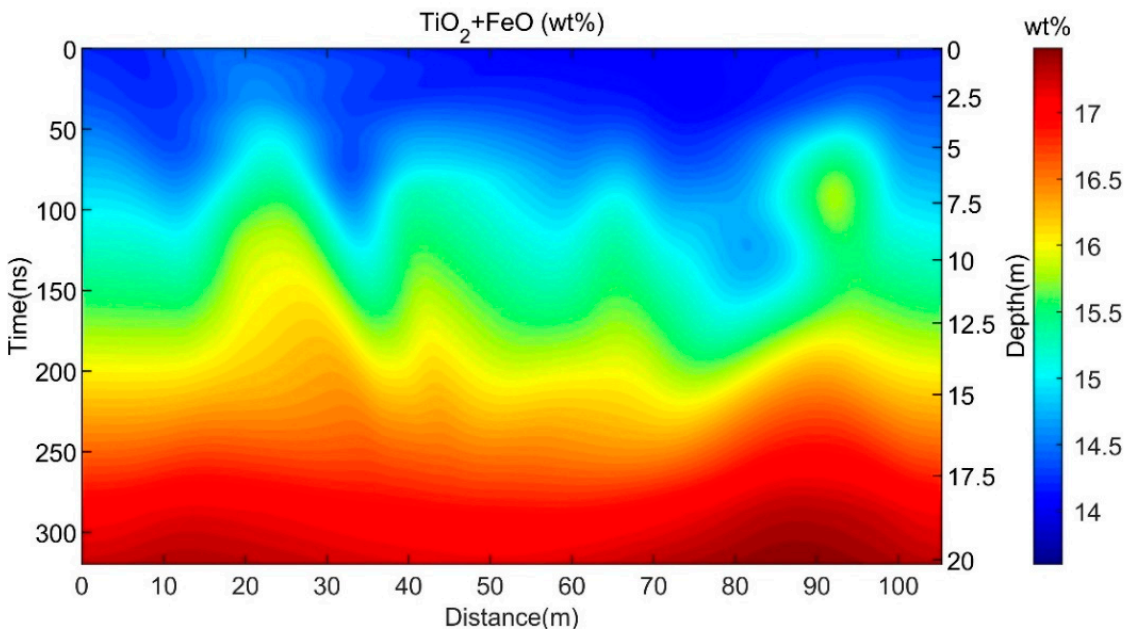

Figure 14. The $\mathrm{TiO}_{2}$ and $\mathrm{FeO}$ content of lunar regolith.

In Figure 10, the points with different velocities distribute in different layers: $0.15 \mathrm{~m} / \mathrm{ns}$ seem to be a watershed; the points whose velocities are bigger than $0.15 \mathrm{~m} / \mathrm{ns}$ are mainly distributed in $0-150 \mathrm{~ns}$ zone; the points whose velocities are smaller than $0.15 \mathrm{~m} / \mathrm{ns}$ are mainly distributed in 150-320 ns zone; this indicates the clear property difference between these two parts which can also be seen from Figures 11-14. These phenomena also correspond to the characteristic of radargram that 0-150 ns zone is of high weathering degree with few rock reflections and 150-320 ns zone is of low weathering degree with strong reflections.

Figures 11-14 show that the velocity descends as the depth increases, and the opposite varieties for velocity and relative permittivity are due to (4). In these figures, we can also find that there is a fluctuation at about $250 \mathrm{~ns}$ after position of $80 \mathrm{~m}$, which also corresponds to the analysis in which the material and horizontal structure changes at a position of $80 \mathrm{~m}$; the material about $250 \mathrm{~ns}$ in Region 3 does not extend to Region 4.

Previous research shows that the relative permittivity of lunar basalt is about 8 [24], so based on the velocity and relative permittivity structure we obtained, we think the region we researched (0-320 ns) is mainly the lunar regolith. The depth coordinates in Figures 9-14 are determined using average root-mean-square velocity of all traces; so the thickness of the lunar regolith is more than $20 \mathrm{~m}$. In addition, the $\mathrm{TiO}_{2}$ and $\mathrm{FeO}$ content in the bottom of Figure 14 is about $17.5 \mathrm{wt} \%$, which is close to the shallow lava flow observed in $[3,4]$, so we speculate that the surface of the basalt is within $25 \mathrm{~m}$ in depth. 


\section{Discussion}

In this section, the estimation errors of two-way reflected time and velocity are discussed. Take the first slice in Figure $8 b$ for instance, a 3D surface plot can be computed, which is shown in Figure 15a. The maximum value in this figure indicates the two-way reflected time and velocity of this hyperbola. However, some error will generate from the noise interference and incomplete hyperbola; the correct point is probably in a region; a flat at an amplitude of 0.8 is selected to split out this region. Two profiles, which are the red rectangle and black rectangle in Figure 15, are extracted out to compute the error intervals of velocity and reflected time, respectively. The two intersections of the blue curve and gray line around the maximum value are selected to compose the error limit.

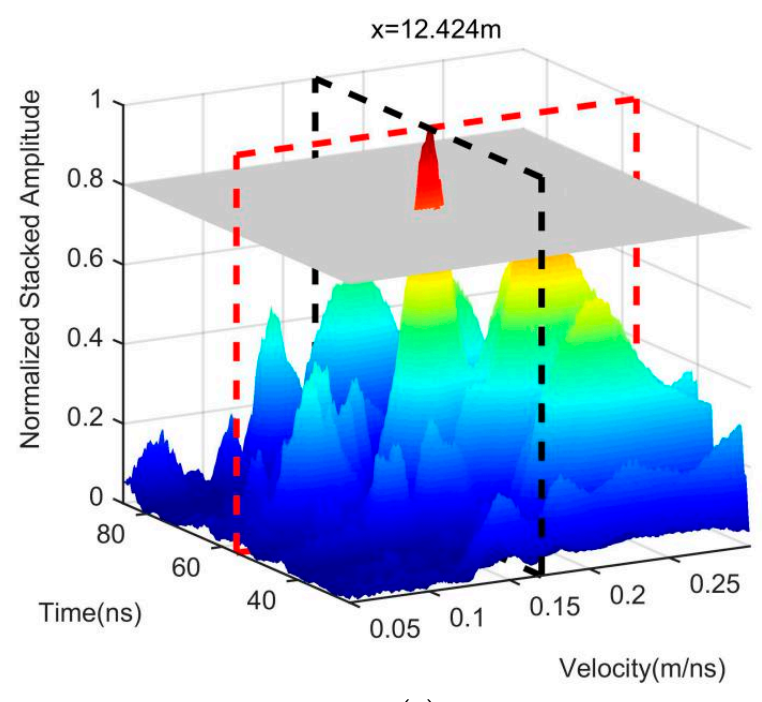

(a)

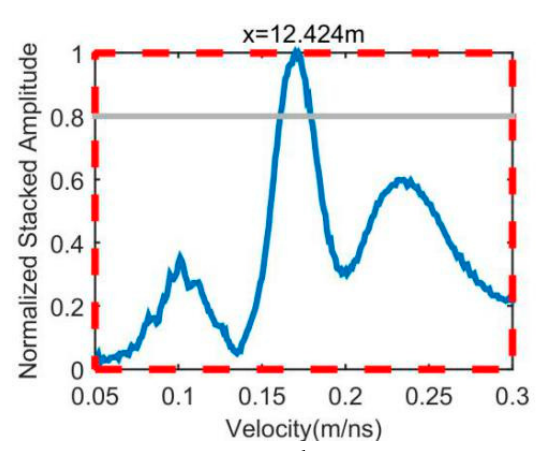

(b)

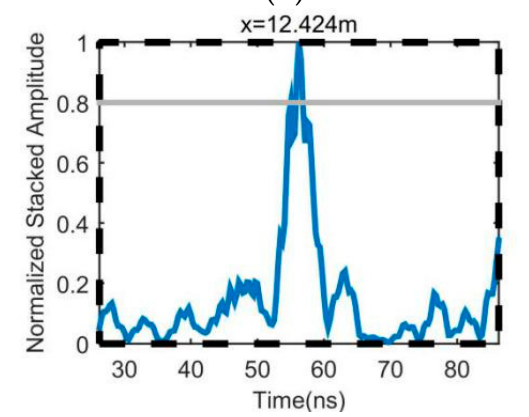

(c)

Figure 15. Error computation. (a) 3D surface plot of the first slice in Figure 8b. (b) The profile of in the red rectangle of (a). (c) The profile of in the black rectangle of (a).

In Figure 15, the error ranges of velocity and two-way reflected time are $0.161-0.179 \mathrm{~m} / \mathrm{ns}$ and $55.8-56.84 \mathrm{~ns}$, respectively. So, the real velocity and time may be within these two ranges. A maximum relative error (MRE) can be adopted to quantitatively describe the error:

$$
\left\{\begin{array}{l}
M R E_{v}=\left|\max \left(v-v_{\text {ran }}\right)\right| / v \\
M R E_{t}=\left|\max \left(t-t_{\text {ran }}\right)\right| / t
\end{array}\right.
$$

where $v$ and $t$ are the estimated values using $3 \mathrm{D}$ velocity spectrum, $v_{\text {ran }}$ and $t_{\text {ran }}$ represent the error ranges. So, the $M R E_{v}$ and $M R E_{t}$ in Figure 13 are $5.29 \%$ and $1.05 \%$, respectively.

Subsequently, the errors of all hyperbolas are computed. The average MREs of time and velocity are $0.68 \%$ and $7.99 \%$, respectively. It is clear that the error of two-way reflected time can be omitted, which is far less than the error of velocity. Furthermore, we computed the distributions of velocities and error ranges in horizontal and vertical directions which are shown in Figure 16. 
(a)

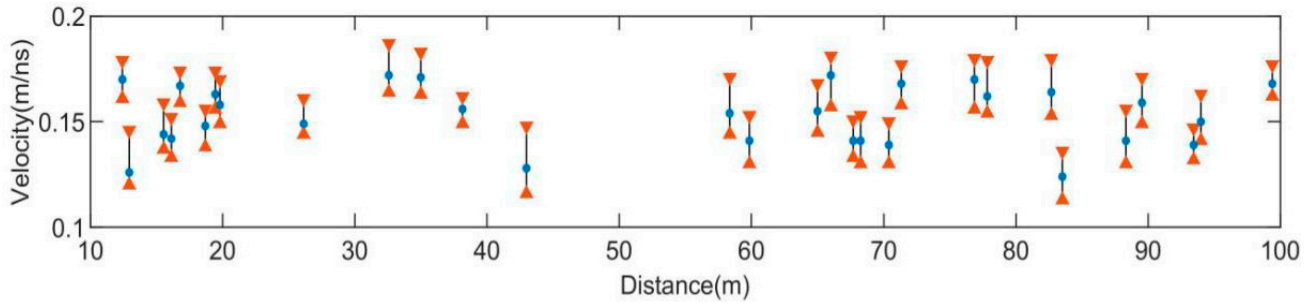

(b)

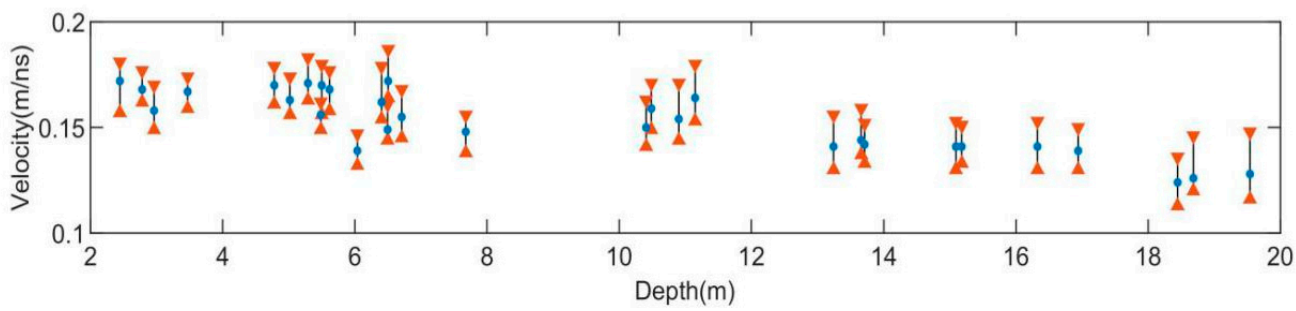

Figure 16. Distributions of velocities and error ranges. (a) Velocities and error ranges with distance. (b) Velocities and error ranges with depth. The triangular points represent the error ranges; the blue dots represent the estimated velocities.

\section{Conclusions}

This paper applies the 3D velocity spectrum to property analysis of lunar penetrating radar data from Chang'E-4. The result shows that 3D velocity spectrum can automatically search for hyperbolas; the maximum value along velocity axis with a soft threshold function can provide the horizontal position, two-way reflected time and velocity of each hyperbola. Based on the estimated velocities of 30 hyperbolas, the subsurface properties' structures are obtained, including velocity, relative permittivity, density, and content of $\mathrm{FeO}$ and $\mathrm{TiO}_{2}$. Combining these properties and the radargram, we can conclude that $0-12 \mathrm{~m}$ is the fine-grained regolith; $12-20 \mathrm{~m}$ is the coarse regolith of different sources; the thickness of the lunar regolith is more than $20 \mathrm{~m}$; the surface of the shallow basalt is within $25 \mathrm{~m}$ in the depth. Importantly, the subsurface material and structure at the Chang'E-4 landing site vary both vertically and horizontally, so the simple horizontal stratigraphic division is not appropriate within the zone of 25-300 ns. Finally, the estimation error is discussed, the errors of horizontal position and two-way reflected time are small which can be omitted; the average maximum relative error of velocity is $7.99 \%$. the distributions of velocities and error ranges in horizontal and vertical directions are obtained.

Author Contributions: Conceptualization, X.F., C.L., Z.Z. and J.L.; data curation, Z.D.; formal analysis, Z.D., H.Z., C.L., Z.Z. and W.L.; funding acquisition, X.F. and W.L.; investigation, H.Z., Z.Z. and J.L.; methodology, Z.D., X.F. and H.Z.; project administration, X.F., J.L. and W.L.; resources, C.L., Z.Z., J.L. and W.L.; software, H.Z.; supervision, C.L.; validation, Z.Z.; visualization, Z.D.; writing—original draft, Z.D. and H.Z.; writing-review and editing, X.F. All authors have read and agreed to the published version of the manuscript.

Funding: This work was funded by Science and Technology on Near-Surface Detection Laboratory under Grant 6142414180911, and the Technology Development Program of Jilin Province: Suppression of Polarization Accompanying Interference for Full-Polarimetric Ground Penetrating Radar under Grant 20180101091JC, and the Fundamental Research Funds for the Central Universities.

Conflicts of Interest: The authors declare no conflict of interest.

\section{References}

1. Ling, Z.; Qiao, L.; Liu, C.; Cao, H.; Bi, X.; Lu, X.; Zhang, J.; Fu, X.; Li, B.; Liu, J. Composition, mineralogy and chronology of mare basalts and non-mare materials in Von Kármán crater: Landing site of the Chang'E4 mission. Planet. Space Sci. 2019, 179, 104741. [CrossRef]

2. Liu, J.; Ren, X.; Yan, W.; Li, C.; Zhang, H.; Jia, Y.; Zeng, X.; Chen, W.; Gao, X.; Liu, D.; et al. Descent trajectory reconstruction and landing site positioning of Chang'E-4 on the lunar farside. Nat. Commun. 2019, 10, 4229. [CrossRef] 
3. Qiao, L.; Ling, Z.; Fu, X.; Li, B. Geological characterization of the Chang'e-4 landing area on the lunar farside. Icarus 2019, 333, 37-51. [CrossRef]

4. Huang, J.; Xiao, Z.; Flahaut, J.; Martiont, M.; Head, J.; Xiao, X.; Xie, M.; Xiao, L. Geological characteristics of Von Kármán crater, northwestern South Pole-Aitken Basin: Chang'E-4 landing site region. J. Geophys. Res. Planets 2018, 123, 1684-1700. [CrossRef]

5. Di, K.; Liu, Z.; Liu, B.; Wan, W.; Peng, M.; Li, J.; Xie, J.; Jia, M.; Niu, S.; Xin, X.; et al. Topographic Analysis of Chang'E-4 Landing Site Using Orbital, Descent and Ground Data. In Proceedings of the International Archives of the Photogrammetry, Remote Sensing and Spatial Information Sciences, Enschede, The Netherlands, 10-14 June 2019; pp. 1383-1387.

6. Hu, X.; Ma, P.; Yang, Y.; Zhu, M.; Jiang, T.; Lucey, P.G.; He, Z. Mineral abundances inferred from in situ reflectance measurements of Chang'E-4 landing site in South Pole-Aitken basin. Geophys. Res. Lett. 2019, 46, 9439-9447. [CrossRef]

7. Xiao, L.; Zhu, P.; Fang, G.; Xiao, Z.; Zou, Y.; Zhao, J.; Zhao, N.; Yuan, Y.; Qiao, L.; Zhang, X.; et al. A young multilayered terrane of the northern Mare Imbrium revealed by Chang'E-3 mission. Science 2015, 347, 1226-1229. [CrossRef] [PubMed]

8. Fa, W.; Zhu, M.; Liu, T.; Plescia, J.B. Regolith stratigraphy at the Chang'E-3 landing site as seen by lunar penetrating radar. Geophys. Res. Lett. 2015, 42, 10,179-10,187. [CrossRef]

9. Dong, Z.; Fang, G.; Ji, Y.; Gao, Y.; Wu, C.; Zhang, X. Parameters and structure of lunar regolith in Chang'E-3 landing area from lunar penetrating radar (LPR) data. Icarus 2017, 282, 40-46. [CrossRef]

10. Feng, J.; Su, Y.; Ding, C.; Xing, S.; Dai, S.; Zou, Y. Dielectric properties estimation of the lunar regolith at CE-3 landing site using lunar penetrating radar data. Icarus 2017, 284, 424-430. [CrossRef]

11. Su, Y.; Fang, G.; Feng, J.; Xing, S.; Jing, Y.; Zhou, B. Data processing and initial results of Chang'E-3 lunar penetrating radar. Res. Astron. Astrophys. 2014, 14, 1623-1632. [CrossRef]

12. Wang, K.; Zeng, Z.; Zhang, L.; Xia, S.; Li, J. A compressive-sensing-based approach to reconstruct regolith structure from lunar penetrating radar data at the Chang'E-3 landing site. Remote Sens. 2018, 10, 1925. [CrossRef]

13. Jia, Z.; Liu, S.; Zhang, L.; Hu, B.; Zhang, J. Weak Signal Extraction from Lunar Penetrating Radar Channel 1 Data Based on Local Correlation. Electronics 2019, 8, 573. [CrossRef]

14. Zhang, L.; Zeng, Z.; Li, J.; Lin, J.; Hu, Y.; Wang, X.; Sun, X. Simulation of the Lunar Regolith and Lunar-Penetrating Radar Data Processing. IEEE J. Sel. Top. Appl. Earth Obs. Remote Sens. 2018, 11, 655-663. [CrossRef]

15. Zhang, J.; Zeng, Z.; Zhang, L.; Lu, Q.; Wang, K. Application of Mathematical Morphological Filtering to Improve the Resolution of Chang'E-3 Lunar Penetrating Radar Data. Remote Sens. 2019, 11, 524. [CrossRef]

16. Lai, J.; Xu, Y.; Zhang, X.; Tang, Z. Structural analysis of lunar subsurface with Chang'E-3 lunar penetrating radar. Planet. Space Sci. 2016, 120, 96-102. [CrossRef]

17. Zhang, L.; Zeng, Z.; Li, J.; Huang, L.; Huo, Z.; Wang, K.; Zhang, J. Parameter Estimation of Lunar Regolith from Lunar Penetrating Radar Data. Sensors 2018, 18, 2907. [CrossRef] [PubMed]

18. Yilmaz, Ö. Seismic Data Analysis, 3rd ed.; Society of Exploration Geophysicists: Tulsa, Oklahoma, 2001.

19. Feng, X.; Sato, M. Pre-stack migration applied to GPR for landmine detection. Inverse Probl. 2004, 20, S99-S115. [CrossRef]

20. Fisher, E.; McMechan, G.A.; Annan, A.P. Acquisition and processing of wide-aperture ground-penetrating radar data. Geophysics 1992, 57, 495-504. [CrossRef]

21. Greaves, P.J.; Lesmes, D.P.; Lee, J.M.; Toksoz, M.N. Velocity variations and water content estimated from multi-offset, ground-penetrating radar. Geophysics 1996, 61, 683-695. [CrossRef]

22. Leparoux, D.; Gibert, D.; Côte, P. Adaptation of prestack migration to multi-offset ground-penetrating radar (GPR) data. Geophys. Prospect. 2001, 49, 374-386. [CrossRef]

23. Hu, B.; Wang, D.; Zhang, L.; Zeng, Z. Rock Location and Quantitative Analysis of Regolith at the Chang'e 3 Landing Site Based on Local Similarity Constraint. Remote Sens. 2019, 11, 530. [CrossRef]

24. Sen, P.N.; Scala, C.; Cohen, M.H. A self-similar model for sedmentary rocks with application to the dielectric constants for fused glass beads. Geophysics 1981, 46, 781-795. [CrossRef]

(C) 2020 by the authors. Licensee MDPI, Basel, Switzerland. This article is an open access article distributed under the terms and conditions of the Creative Commons Attribution (CC BY) license (http://creativecommons.org/licenses/by/4.0/). 